Marquette University

e-Publications@Marquette

Physics Faculty Research and Publications

Physics, Department of

$6-1-2007$

\title{
Micromagnetics of the Domain Wall Mobility in Permalloy Nanowires
}

Andrew Kunz

Marquette University, andrew.kunz@marquette.edu

Accepted version. IEEE Transactions on Magnetics, Vol. 43, No. 6 (June 2007): 2944 - 2946. DOI. () 2007 Institute of Electrical and Electronics Engineers (IEEE). Used with permission. 


\section{Marquette University}

\section{e-Publications@Marquette}

\section{Physics Faculty Research and Publications/College of Arts and Sciences}

This paper is NOT THE PUBLISHED VERSION; but the author's final, peer-reviewed manuscript. The published version may be accessed by following the link in the citation below.

IEEE Transactions on Magnetics, Vol. 43, No. 6 (xxxx): 2944-2946. DOI. This article is C) Institute of Electrical and Electronics Engineers (IEEE) and permission has been granted for this version to appear in e-Publications@Marquette. IEEE does not grant permission for this article to be further copied/distributed or hosted elsewhere without the express permission from IEEE.

\section{Micromagnetics of the Domain Wall Mobility in Permalloy Nanowires}

\section{Andrew Kunz}

Department of Physics, Marquette University, Milwaukee, WI

\section{Keywords}

Micromagnetics, Nanowires, Magnetic domain walls, Wire, Magnetic domains, Magnetic devices, Spatial resolution, Magnetic fields, Equations, Computational modeling

\section{Abstract: \\ The domain wall mobility in long permalloy nanowires with thicknesses of 2-20 nm and widths of 50- $200 \mathrm{~nm}$ has been simulated. The domain wall is driven into motion by an external magnetic field and the average wall mobility is calculated after the wall has traveled 2.5 mum along the wire. The results were obtained using the three-dimensional dynamic Landau-Lifshitz equation. We find that the domain wall mobility decreases linearly up to the critical field called the Walker field. The decreasing wall mobility is related to the decrease in the dynamic domain wall length as the applied field is increased. The value of the critical field is dependent on the thickness and width of the wire. At the critical field the mobility decreases by an order of magnitude. Above the Walker field the average mobility remains}


relatively constant for all driving fields, while the instantaneous mobility shows regions of high mobility with long periods of almost no mobility. For large applied fields the domain wall velocity can be large even though the average mobility is low.

\section{SECTION I.}

\section{Introduction}

The increase in computer speed and power combined with the shrinking dimensions of new technological devices makes it viable to use micromagnetic simulation to accurately explore the behavior of a wide variety of physical magnetic structures at low cost. In nanowire devices the experimental analysis of domain wall motion is difficult because techniques are not able to jointly examine the wall structure and dynamics; instead relying on average values ${ }^{[1], ~[2] . ~ T h e ~ l a c k ~ o f ~ s u f f i c i e n t ~}$ resolution has led to conflicting results in the literature ${ }^{[3], ~[4]}$. The combination of nanometer spatial resolution and concurrent picosecond temporal resolution makes micromagnetic simulation an ideal method for studying the field driven domain wall motion in a magnetic nanowire.

Domain wall motion in a magnetic nanowire has potential for application in many important technological areas including magnetic recording, magnetoresistive sensing, and digital devices for logic operations ${ }^{[5]-[6][7]}$. In each of these applications information is carried by a domain wall traveling in the wire. The domain wall must be controllably moved both quickly and reliably in the wire if the magnetic technologies are to be competitive with current devices. However, the domain wall speed is often slow depending on the cross-sectional area of the nanowires ${ }^{[8]}$.

Wall mobility is typically reported as a single value but we find that the domain wall mobility, shown in Fig. 1, decreases linearly as the driving field increases up to the critical Walker field. The reduction in wall mobility in this region is about $25 \%$. The Walker field depends on the wires cross-sectional area. At the Walker field the mobility abruptly decreases and then remains essentially constant as the applied field increases, as shown in the inset of Fig. 1. For large field strengths, the domain wall velocity can be quite large even though the mobility is small. 


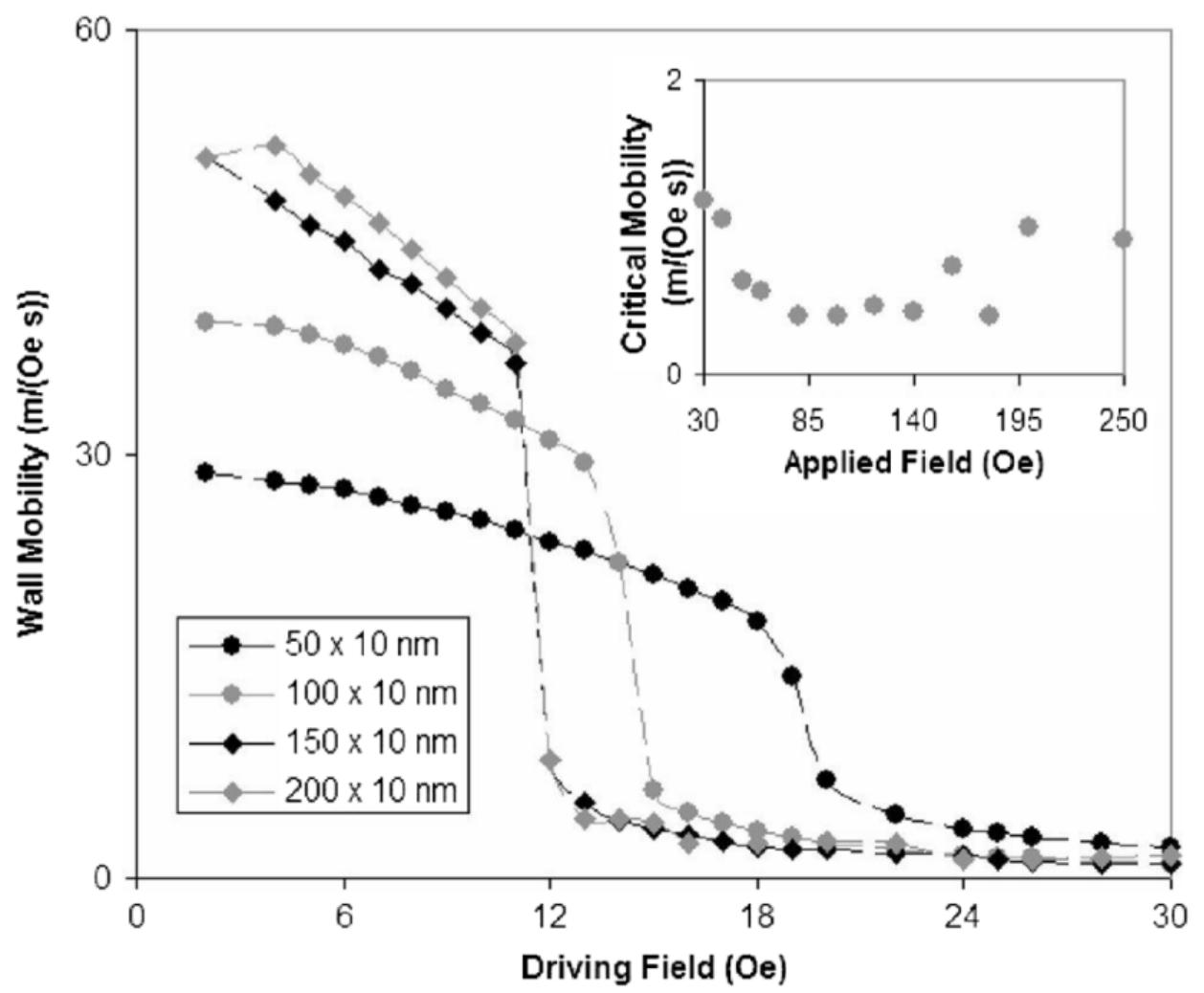

Fig. 1. Domain wall mobility as a function of driving field for four nanowire widths. The mobility decreases linearly with applied field up to the critical field. The inset shows the high field mobility for the $100 \times 10-\mathrm{nm}$ crosssection wire. For large fields the mobility remains nearly constant and the domain walls move with large speeds.

\section{SECTION II.}

\section{Simulation Details}

Each simulated nanowire is $5 \mu \mathrm{m}$-long with cross-sectional areas of width $50-200 \mathrm{~nm}$ and thickness 2$20 \mathrm{~nm}$. To carry out the micromagnetic simulations [9] the nanowire is discretized into identical cubes of uniform magnetization between 2-5 nm (depending on the wire thickness). When possible, the effects due to the discretization are checked showing no qualitative or quantitative difference in the results. The small discretization size allows for three-dimensional modeling in wires greater than 5-nm thick. 


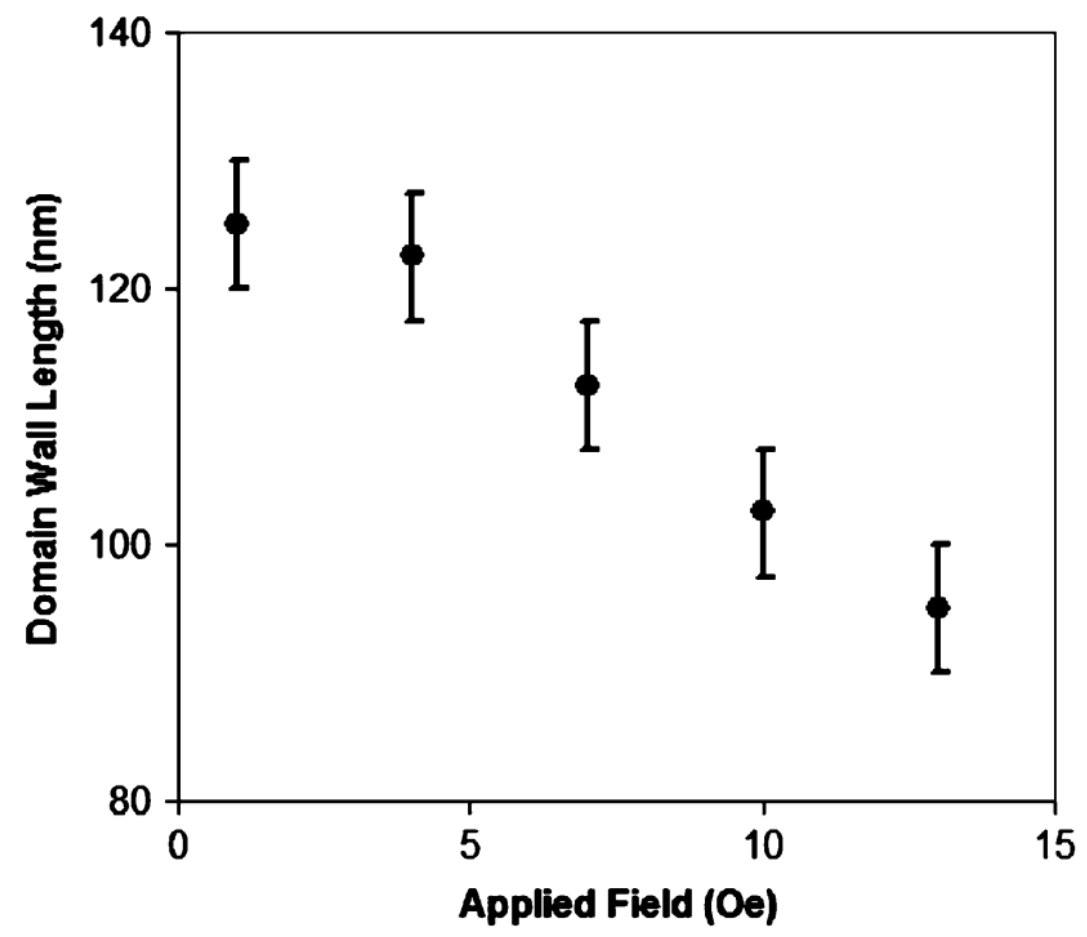

Fig. 2. Domain wall length in a $100 \times 5-\mathrm{nm}$ cross-section wire. The dynamic domain wall length decreases linearly up to the value of the critical field. The decrease in length corresponds to a similar decrease in wall mobility below the critical field. The uncertainly arises from the size of the discretization used in the simulation.

Micromagnetic simulation follows the motion of each magnetic moment $\vec{m} \vec{i}$, in the total magnetic field $\vec{H}_{i}$, by integrating the three-dimensional Landau-Lifshitz equation of motion

$$
\frac{\partial \vec{m}_{i}}{\partial t}=-\gamma\left(\vec{m}_{i} \times \vec{H}\right)-\frac{\alpha \gamma}{M_{s}} \vec{m}_{i} \times\left(\vec{m}_{i} \times \vec{H}_{i}\right)
$$

where $\gamma=17.6 \times 10^{6} \mathrm{Oe}^{-1} \mathrm{~s}^{-1}$. The first term on the right hand side of $(1)$ describes the precessional motion of the magnetic moment about the local field while the second term removes energy from the moments allowing them to rotate into the direction of the local field. The total field $H$ includes the applied field, the long-range magnetostatic field (due to all of the moments in the wire,) and the nearest neighbor exchange field with the parameters $M_{S}=800=800 \mathrm{emu} / \mathrm{cm}^{3}$ and $\mathrm{A}=1.3 \times 10^{-6}$ $\mathrm{erg} / \mathrm{cm}$ respectively for permalloy. No crystalline anisotropy was used in this simulation.

A physical interpretation of the magnetization dynamics depends on the value of the phenomenological damping parameter $\alpha$. For the results presented here $\alpha=0.008$. Previous work has shown how the dynamics depend on the value of the damping parameter ${ }^{[10]}$. The numerical integration uses a fourth order predictor-corrector method with a subpicosecond time step. The wire is subject to free boundary conditions on all of its edges. 
A sharp head-to-head domain wall is artificially placed into the nanowire in the simulations. In zero applied field the transition between the two oppositely oriented domains is allowed to relax to the equilibrium domain wall structure. The wall is placed near one end of the wire and moved completely out the other side. Similar to many experimental methods, the average speed of the domain wall is calculated by measuring the transit time across the central $2.5 \mu \mathrm{m}$ region of the wire. This allows over $1 \mu \mathrm{m}$ on each side of the measured motion; eliminating coupling effects of the domain wall with the ends of the wire. The domain wall accelerates when it gets within a few domain wall lengths of the end of the wire. Comparisons of the speed have been made on nanowires up to $20 \mu \mathrm{m}$ in length with no measurable effect on the overall wall speed.

The shape anisotropy of the nanowire predominantly aligns the magnetic moments in the wire parallel to the long axis. In the domain wall the moments deviate from parallel. The domain wall length shown in Fig. 2 is determined by measuring the region where the moments deviate from parallel. The uncertainty in the value of the wall length arises from the size of the discretization step used in the simulation which makes it impossible to determine the exact extent.

\section{SECTION III.}

\section{Results and Discussion}

The speed of the domain wall below the Walker field is a function of the applied field [11]

$$
v=\mu H_{a}
$$

where the mobility

$$
\mu=\left(\frac{\gamma}{\alpha}\right) \Delta
$$

depends on the domain wall length $\Delta$, the spatial extent of the wall measured in the same direction as the length of the wire. The wall speed and length, as shown in Fig. 2, are both functions of the applied field which means $(2)$ is a nonlinear function of the driving field.

The dynamic domain wall length decreases linearly from its equilibrium zero-field length. Energetically it is the Zeeman term $-m \cdot H_{a}$ which depends on the strength of the applied field. The Zeeman energy is linear in the applied field. Therefore it is expected that the wall length will decrease linearly with increasing applied field strength. The effects of the decrease in the domain wall length are seen in Fig. 1 as a decrease in domain wall mobility below the Walker field.

We find that the relative decrease in mobility matches the relative decrease in domain wall length, below the Walker field limit, with each varying up to $25 \%$. 
Fig. 1 shows that the wall mobility depends strongly on the width of the nanowire. Fig. 3 shows the critical mobility (mobility at the critical field) for a variety of nanowire cross sections. For a single wire thickness the mobility depends strongly on the wire width, changing by $30 \mathrm{~m} /(\mathrm{Oe} \mathrm{s})$.

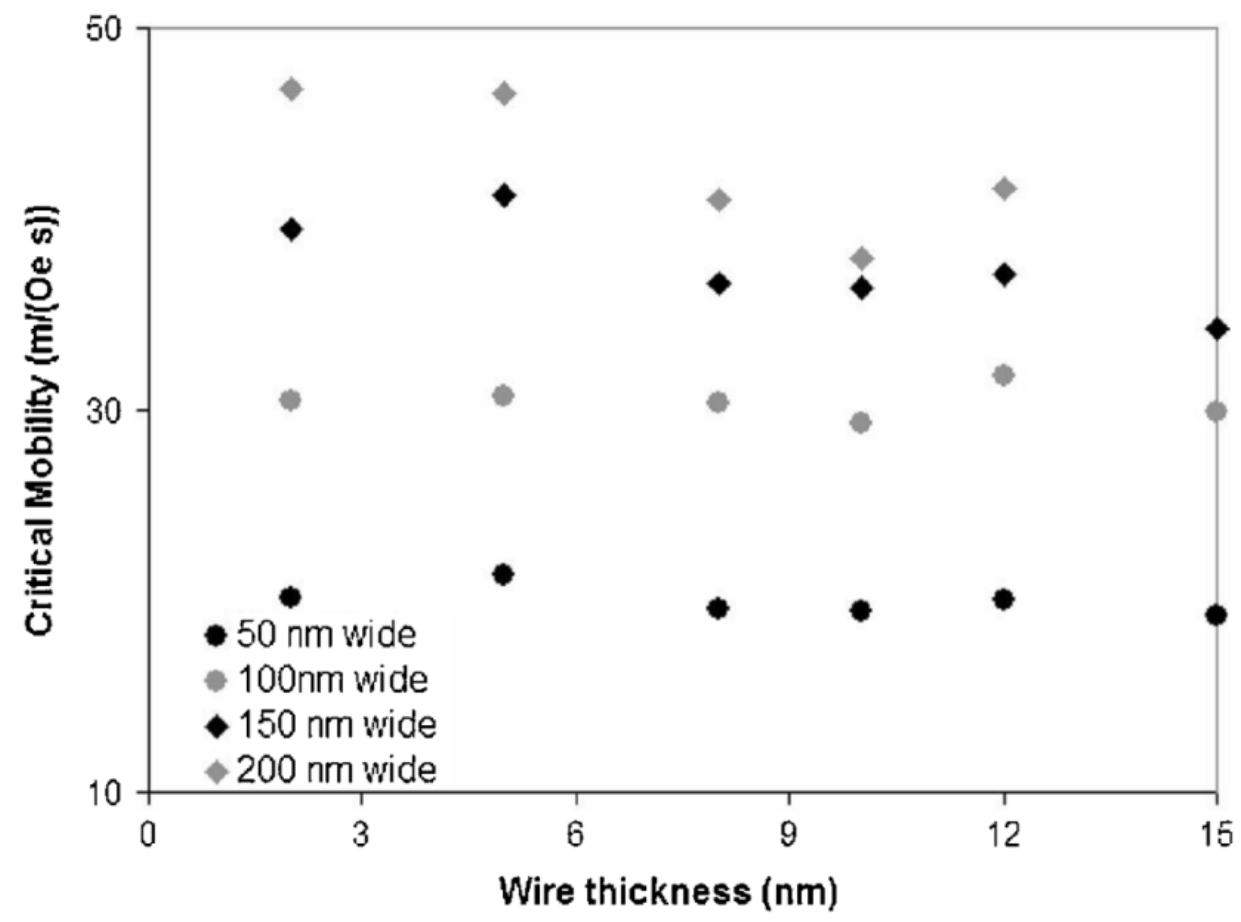

Fig. 3. Critical wall mobility versus wire thickness for four wire widths. In narrow thin wires the mobility remains essentially constant as the thickness is increased. As the wire dimensions increase the domain wall structure can be more complicated which decreases the wall mobility.

Fig. 3 shows that the domain wall mobility is independent of the wire thickness ${ }^{[12]}$, over the range of thicknesses observed as long at the wire width is less than $100 \mathrm{~nm}$. In a thin wire the domain wall is transverse and inspection of the structure shows that the magnetization is uniform throughout the thickness. The domain wall is therefore one-dimensional and its equilibrium structure only depends on the wire width. In Fig. 3, deviations from linearity appear as the wire becomes thicker and wider. This is because there is more domain wall structure as the dimensions of the wire grow.

While the mobility changes significantly below the critical field the inset of Fig. 1 shows that above it the average wall mobility is relatively constant. However, Fig. 4 shows and previous work has shown that the instantaneous mobility is always changing [13]-[14][15]. Above the Walker field a vortex nucleates at a domain wall edge and propagates through the traveling wall. Depending on the presence of these vortices the instantaneous wall mobility can be positive, negative, or essentially zero. The fluctuations in the instantaneous mobility give rise to low average mobility when calculated over large lengths of the wire. The maximum mobility does reach the critical mobility and the wall moves quickly for short periods of time. Fig. 1 shows that the mobility is relatively constant above the critical field. This gives rise to fast domain wall motion for large applied fields. The wall speeds approach the value of the critical velocity found here and in previous work. 


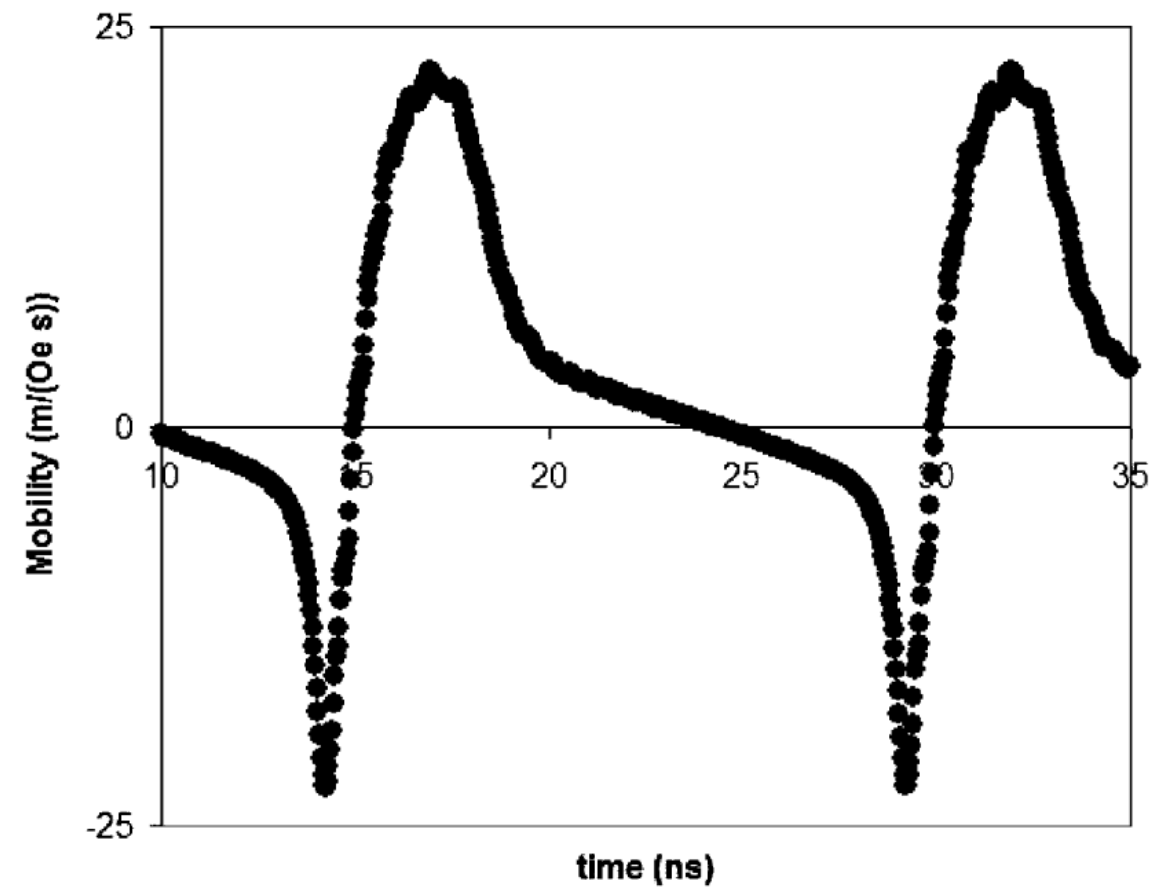

Fig. 4. The instantaneous wall mobility for a $100 \times 10-\mathrm{nm}$ cross-section nanowire when driven by an 18 Oe field (greater than the critical field). The domain wall exhibits both positive and negative mobility, with long time periods where the mobility is essentially zero leading to low average mobility.

\section{SECTION IV.}

\section{Conclusion}

Domain wall mobility in permalloy nanowires has been calculated using micromagnetic simulation for a large range of applied fields and nanowire cross sections. The wall mobility decreases linearly up to the critical field because the dynamic domain wall length also decreases with increasing field strength. The critical mobility is found to be dependent on the width of the wire however. Above the critical field the wall mobility remains constant leading to fast wall motion for large field strengths.

\section{ACKNOWLEDGMENT}

This work was supported by the Marquette University Helen Way Klingler College of Arts and Sciences. The author would like to thank D. Dahlberg for access to the micromagnetic license and B. Kaster, an undergraduate student who has been assisting in the simulations and analysis.

\section{REFERENCES}

${ }^{1}$ G. S. D. Beach, "Dynamics of field-driven domain-wall propagation in ferromagnetic nanowires", Nature Mater., vol. 4, pp. 741-44, 2005. 
2 D. Atkinson, "Magnetic domain wall dynamics in a permalloy nanowire", IEEE Trans. Mag., vol. 39, no. 5, pp. 2663-2665, Sep. 2003.

3 T. Ono, "Propagation of a domain wall in a submicrometer magnetic wire", Science, vol. 284, pp. 46870, 1999.

${ }^{4}$ D. Atkinson, "Magnetic domain-wall dynamics in a submiicrometre ferromagnetic structure", Nature Mater., vol. 2, pp. 85-87, 2003.

${ }^{5}$ E. Saitoh, H. Miyajima, T. Yamaoka, G. Tatara, "Current-induced resonance and mass determination of a single magnetic domain wall", Nature, vol. 432, pp. 203-206, 2004.

${ }^{6}$ I. N. Krivorotov, "Time-domain measurements of nanomagnet dynamics driven by spin-transfer torques", Science, vol. 307, pp. 228-231, 2005.

7 D. A. Allwood, "Magnetic domain-wall logic", Science, vol. 309, pp. 1688-1691, 2005.

${ }^{8}$ A. Kunz, "Simulated domain wall dynamics in magnetic nanowires", J. Appl. Phys., vol. 99, 2006.

${ }^{9}$ A. Kunz, "Simulating the maximum domain wall speed in a magnetic nanowire", IEEE Trans. Magn., vol. 42, no. 10, pp. 3219-3221, Oct. 2006.

${ }^{10}$ N. L. Schryer, L. R. Walker, "The motion of $180^{\circ}$ domain walls in uniform dc magnetic fields", J. Appl. Phys., vol. 45, pp. 5406-21, 1974.

${ }^{11}$ M. Redjdal, J. Guisti, M. F. Ruane, F. B. Humphrey, "Thickness dependent wall mobility in thin permalloy films", J. Appl. Phys., vol. 91, pp. 7457-59, 2002.

12 Y. Nakatani, A. Thiaville, J. Miltat, "Faster magnetic walls in rough wires", Nature Mater., vol. 2, pp. 521-523, 2003.

${ }^{13}$ D. G. Porter, M. J. Donahue, "Velocity of transverse domain wall motion along thin narrow strips", J. Appl. Phys., vol. 95, pp. 6729-6731, 2004.

${ }^{14} \mathrm{~L}$. Thomas, "Oscillatory dependence of current-driven magnetic domain wall motion on current pulse length", Nature, vol. 443, pp. 197-200, 2006. 